\title{
Clustering and Analysis of Dynamic Ad Hoc Network Nodes Movement Based on FCM Algorithm
}

\author{
https://doi.org/10.3991/ijoe.v16i12.16067 \\ Sumaya Hamad ${ }^{(凶)}$, Khattab M. Ali Alheeti \\ University of Anbar, Anbar, Iraq \\ Sumayah.hamadeuoanbar.edu.iq \\ Yossra H. Ali, Shaimaa H. Shaker \\ University of Technology, Baghdad, Iraq
}

\begin{abstract}
Clustering is a major exploratory data mining activity, and a popular statistical data analysis technique used in many fields. Cluster analysis generally speaking isn't just an automated function, but rather reiterated information exploration procedure or multipurpose dynamic optimisation comprising trial and error. Parameters for pre-processing and modeling data frequently need to be modified until the output hits the desired properties. -Data points in fuzzy clustering may probably belong to several clusters. Each data point is assigned membership grades. Such grades of membership reflect the degree to which data points belong to each cluster. The Fuzzy C-means clustering (FCM) algorithm is among the most widely used fuzzy clustering algorithms. In this paper we use this method to find typological analysis for dynamic Ad Hoc network nodes movement and demonstrate that we can achieve good performance of fuzziness on a simulated data set of dynamic ad hoc network nodes (DANET) and how to use this principle to formulate node clustering as a partitioning problem. Cluster analysis aims at grouping a collection of nodes into clusters in such a way that nodes seeing a high degree of correlation within the same cluster, whereas nodes members of various clusters are extremely dissimilar in nature. The FCM algorithm is used for implementation and evaluation the simulated data set using NS2 simulator with optimized AODV protocol. The results from the algorithm 's application show the technique achieved the maximum values of stability for both cluster centers and nodes $(98.41 \%, 99.99 \%)$ respectively and has the highest accuracy (stability) compared to previous methods.
\end{abstract}

Keywords—Cluster Analysis, Fuzzy Clustering, FCM, Ad Hoc Network, NS2 simulator.

\section{Introduction}

Analysis of the cluster oneself isn't a particular algorithm but the general problem that being resolved. This could be accomplished by different algorithms, which vary substantially in their understanding of what makes a cluster and how to identify them efficiently [1][2]. Fuzzy c-means (FCM) clustering has gained significant awareness 
for its special features of many various fuzzy clustering techniques. Since it can aggregate several base clustering approaches with same object set in an unanimity solution; cluster community will have many attractive features like enhanced solution consistency, strong clustering, and information reuse. As for the weak partitioning solution, however, the process still needs to compute and store the product of matrix of membership, which includes complexity of space and time with size of quadratic data. Fuzzy clustering aims at obtaining a versatile partition, in which every element has membership in multiple clusters including values under it $[0,1]$. FCM clustering algorithms are commonly used in low-dimensional data due to their performance and effectiveness. [3][4].

FCM has many benefits, including simple implementation, relatively stable behaviour, information via multi - channel applicability as well as capability of modeling suspicious data; However, spatial-data information couldn't be treated effectively. The problem with data clustering, the system 's dynamic behaviour must be sufficiently excited to make data of the system rich enough [5]. FCM can be viewed as an advanced clustering strategy, allowing each data point to become part of several clusters with different membership degrees. The process works by decreasing the target function [6][7]. The FCM clustering method was successfully implemented in feature analysis, classification model and clustering [8][9].

The purpose of this work is to demonstrate how the fuzziness principle can be utilized to a dynamic ad hoc network node data set. To check the behavior of the simulated data set the algorithm of fuzzy c-means is implemented. In contrast to fast clustering, soft clustering patterns do not need to commit indefinitely to a center of cluster so this enables the objective function to escape from the local extreme. Our goal was not to propose clustering algorithm to show best results as for the functional cost, not one more effective one. Rather we aim to launch the basis for providing extra axiomatic and more naturalistic mechanism for data analyst to control the process of clustering for the context of data exploring. The main stimulus is to offer a collection of rational clusters from the appropriate ones. Even so, the results of experiments indicate that biased algorithm not only appears to significantly increase the successive runs but also appears to produce, on average, reasonable clusters with better index values for validity. We also found less sensitivity to initialization. Such results are known as an appropriate shrinking side effect.

We identify some problems for DANET node movement such as: It is hard to quantify the exploitation as a node moves along the path at different velocities in various directions. The node position is also difficult to find. Higher overhead computing occurs for node movement analysis. The need of node clustering and analysis, node stability is predicted for better performance by proper positioning techniques.

Our contributions are:

i. Building a proposed system using NS2 to identify and monitoring a range of active nodes on communication system of mobility nodes in DANETs utilizing optimized routing protocol Ad hoc On-Demand Distance Vector AODV and generating traffic and mobility sceneries to generate essential information about 
position of node between nodes, its direction, and speed of each node as csv file represents a data set collection step.

ii. Reducing the dimension of data and extracting the position information as a low dimensional data set input for clustering and analysis using FCM algorithm to provide a more precise computation of the cluster membership by doing a full inverse-distance weighting, finding minimizers of objective function so it has been used successfully for DANET clustering applications like military, civilian, and commercial areas. this technique achieves more efficient accuracy (stability) in the result and reduces the time taken for data and/or information extracting from large dataset with the use of continuous values of nodes, as they incorporate more information that could potentially improve subsequent analysis. Improving stability and decreasing the overhead will increase the efficiency of the network.

iii. Compared to other algorithms, the network stability is increased.

The work organized as the following sections: In Section 2, Related Works are presented. In section 3 the Mathematical Model of Fuzzy C-mean are described. Section 4 explains the detailed of the Methodology of the work. Section 5 describes the Experiment results and Discussion. Finally, Section 6 outlines the Conclusions and Future Works.

\section{Related Works}

\subsection{Using FCM technique}

There have been several proposals for the election of FCM algorithm in Ad-hoc networks. A Fuzzy Cluster Mean (FCM)-based clustering approach is proposed for WSNs by W. Zhenhua and et al. This is a new clustering method first to create the clusters, and afterwards pick the head $(\mathrm{CH})$ of the cluster. This FCM-based strategy has strong features of fast clustering, reducing the consumption of energy and being implemented in various modes of transmitting data. In simulations the accuracy and feasibility are verified. The energy efficiency is demonstrated to be higher than equivalent clustering algorithms [10]. Instead, R. Dutta and et al. introduced a new lowenergy adaptive, unequal clustering protocol using Fuzzy c-means in wireless sensor networks (LAUCF), an unfair size of clustering paradigm for network arrangement based on the FCM clustering algorithm which could makes energy dissipation more uniform within the headnodes of the cluster, hence rising the lifespan of the network [8]. G. Abdulsahib and et al. investigate the consequence of using the ad hoc network clustering technique, so how will the strategy improve savings on resources and reduce time delays. Also, it explains the clustering, structure of cluster, method of cluster linking, and various algorithms used for selection of cluster head and their impact on MANETs. Additionally, the study measures effectiveness of MANETs to demonstrate the impact of clustering on ad-hoc networking efficiency, thus a cluster-based routing protocol (CBRP) has been used that will be viewed one of the routing proto- 
cols for clustering. The CBRP is evaluated by comparing to other routing protocol types, like AODV, DSR, and DSDV, and none of them has used the clustering technique. Results are inferred and analyzed to illustrate the benefits and drawbacks of using clustering in MANETs [11]. While J. Gu and et al introduce new clustering algorithm, named sparse learning based fuzzy c-means (SL_FCM). Initially, in order to decrease the computational sophistication of the SR-based FCM process, much of the energy of the discriminating function acquired by resolving an SR model should be reserved and the rest is removed. In this method a few repetitive data (i.e. similarity between samples from the various groups) can also be eliminated in the discriminant feature, which could also increase the efficiency of the clustering. In addition, the current location of valid values is also used as a discriminant feature to redefine the distance between the sample and the clustering center in SL FCM to further enhance the clustering performance. The weighted distance in SL FCM will enhance sample similarities from the same class and sample differences from different classes, thus enhancing the clustering effect. However, as the dimensions of each sample 's stored discriminant feature differ, they utilize set operations to define the distance and cluster center in SL FCM [4]. New fuzzy c-means (FCM) clustering algorithm was introduced with random projection by $\mathrm{M}$. Ye and et al... empirical tests prove how the new algorithm not just retains precision of the classic clustering with FCM, but its more too effective clustering than original and clustering with decomposition of single values. Around the same time a new method of cluster ensemble with random projection based on FCM clustering is suggested. The innovative agglomeration technique could be effective to calculate a spectral embeddings of data with representation based on centers of cluster, which scales with data size linearly [3]. D. Wang and et al. suggest an original two-phase approach to designing and characterizing fuzzy rule-based models in which the FCM algorithm begins with fuzzy sets [12]. A proposed method is named as FCM-Q LEACH-VANET presented by T. Mamatha and P. Aishwarya. Road side unit (RSU) link to base station ( BS) was performed by using IEEE 802.11p protocol to minimize the transfer time from Source (SN) to BS [13].

\subsection{Using other techniques}

Also there have been several proposals for using clustering technique in Ad-hoc networks. Z. Y. Rawashdeh and S. M. Mahmud develop a new clustering technique suitable for the VANET highway setting, for improving network topology stability. This technique utilizes the difference in speed as a parameter to establish a relatively stable arrangement of clusters. A new multimetric algorithm for cluster-head elections was also developed [14]. While C. Konstantopoulos and et al. introduce a novel algorithm for the clustering. The basis of the algorithm is a scheme that precisely predicts each mobile host 's mobility based on its neighborhood stability. This knowledge is then used to build each cluster from hosts that will remain neighbors for long enough, ensuring clusters that are highly resistant to host mobility are created. They use demonstrably good knowledge theoretical techniques to estimate potential host mobility, which allow on-line learning of a robust probabilistic model for current host mobility [15]. Also, Y. Zhang and et al. propose a distributed group mobility adaptive 
(DGMA) clustering algorithm for mobile ad hoc networks ( MANETs) based on a revised group mobility metric, linear distance based spatial dependency (LDSD), derived from the linear movement distance of a node instead of its instantaneous velocity and direction [16]. S. R. Valayapalayam Kittusamy and other authors Present a novel Cluster Structure and Cluster Head $(\mathrm{CH})$ Election Algorithm suitable for VANETs. The proposed adaptive weighted clustering protocol (AWCP) groups the random nodes, and then the optimal $\mathrm{CH}$ is achieved through network parameter optimisation. An advanced algorithm called the enhanced whale optimization algorithm (EWOA) is implemented for optimization purposes. For each vehicle in a trusted clustering model its movement is analyzed with identified speed and position by the vehicle network mobility routing protocol. The AWCP-EWOA model being proposed analyzes the distance between the trusted vehicle node and RSU [17]. An improved multi-parameter weighted clustering algorithm called TCWCA has been developed by L. Shan, L. Zhang to improve the clustering efficiency of wireless networks. . The algorithm takes into consideration the calculation factors of node degree, internode distance, node mobility, node energy, and clustering process association increase in network topology. Additionally, the algorithm applies increments of the regional topology association of network nodes to the weighting factor calculation process [18]. Instead, M. Ren and et al. are developing a new dynamic mobility and stabilitybased clustering system for urban area scenarios. The scheme proposed applies the moving path of the vehicle, the relative location and the lifetime estimate relation [19]. S. Pathak, S. Jain had suggested a prioritized weighted clustering algorithm for MANETs that operates on three strategies for reducing updates in the cluster head and cluster overhead. The first step helps to decide the cluster head dynamically based on their initial priorities within the cluster of neighbor nodes. The second and third step help to automatically pick the new cluster head in cluster maintenance and without delay when the remaining battery power of the old cluster head exceeds the minimum threshold value or in the absence of cluster head [20]. Also, as they before having presented an optimized stable clustering algorithm, which would provide greater network stability by minimizing cluster head changes and reducing overhead clustering. A new node is added in the proposed algorithm which acts as a backup node within the cluster. Such a backup node serves as the head of the cluster, when the real head of the cluster steps out (or died). Later the head of the cluster re-elects a new node for backup [21]. A new algorithm based on weight, proposed by A. Karimi and others not only use their own features to assess the node 's weight, but also consider the direct effect of adjacent node feature. This specifies the weight of virtual node links, and the impact of weights on the final weight of the node. By using this technique, the maximum weight is allocated to the best choices for being the heads of the cluster and the collection of nodes increases in accuracy [22]. M. Chatzidakis, with S. Hadjiefthymiades propose a clustering scheme that produces clusters that are sufficiently stable to allow confidence information to propagate, initially through the cluster and, eventually, through the entire network. They also suggest a trust scheme that assigns and updates a trust value to each network node, thus exposing the malicious nodes and disseminating the knowledge across the network [23]. P. Basu and et al. suggest a distributed clustering algorithm, MOBIC, based on the use of this mobility measure for 
cluster head selection, and show that this results in a more stable cluster creation than the model of the well-known Lowest-ID clustering algorithm [24]. Henceforth an appropriate technique should be planned that easily responds to the changes in topology. Initially, the VANET clustering problem (CP) is developed into a problem of dynamic optimization. Next, it is proposed that an optimization algorithm called Vehicular Genetic Bee Clustering (VGBC) based on honeybee algorithm and genetic algorithm properties solve the CP in VANETs. Individuals (bees) represent a practical clustering structure in VGBC, and their fitness is calculated based on load balance and stability. M. Ahmad and others are proposing a methodology that fuses the properties of the genetic algorithm and the honey bee algorithm [25]. As they suggested before, honeybee algorithm-based clustering generates clusters efficiently with less resources such as usage of energy and bandwidth. A node is selected as a cluster head based on degree of node, behavior of the neighbor, direction of mobility, speed of mobility and remaining energy. The proposed technique inspired by the foraging behavior of honey bees gives effective and stable cluster forming due to the productive existence of the bees and the consideration of the maximum parameter [26]. K. A. Awan and others present a trust-based clustering framework that enables clusters to recognize a trusted $\mathrm{CH}$. The novel features incorporated in the proposed technique include trust-based selection of $\mathrm{CH}$ which includes a node 's knowledge, reputation and experience. A backup head is often calculated by an analysis of the trust of each node in a cluster. The main advantage of using clustering trust is detecting malicious and compromised nodes. Recognition of these nodes helps eliminate the possibility of invalid knowledge [27]. Considering the high efficiency of clustering methods among the routing algorithms S. A. Sharifi and S. M. Babamir produce a new clustering method and considering fine performance of Evolutionary Algorithms (EAs) in finding suitable head clusters, they present a different EA-based method called ICA (Imperialist Competitive Algorithm) by numerical coding. By thinking about particular conditions of a MANET and predicting the direction of mobility of nodes, they avoid further reclustering leading to reduced overload [28]. While a novel approach of dynamic clustering mechanism with nondeterministic simulated (DCHA) technique is implemented by R. Sundar and A. Kathirvel to avoid loss of path and to create a stable connection between the source and the destination. Dynamic clusters are arranged along the path based on the minimum local and the minimum global of mobile nodes [29]. M. Ni and et al. are proposing a mobility prediction-based clustering (MPBC) scheme for high mobility node ad hoc networks where a node can alter the associated cluster head $(\mathrm{CH})$ many times during its connection lifetime. The suggested clustering framework involves an initial clustering process and a cluster maintenance phase. The Doppler shifts associated with regularly exchanged Hello packets among neighboring nodes are used to estimate their relative velocities, and the estimation results are used in MPBC as basic information. The nodes with the smallest relative mobility in their neighborhoods are selected as $\mathrm{CHs}$ in the initial clustering stage. Mobility prediction techniques are implemented in the cluster maintenance stage to manage the various problems caused by node motions, such as potential association losses to current $\mathrm{CHs}$ and $\mathrm{CH}$ position changes, to increase the lifespan of the connection and provide more stable clusters. An analytical model is constructed to determine the upper and lower 
limits of the average lifespan of the connection and determine the MPBC average rate of change in the relationship [30].

\section{$3 \quad$ Fuzzy C-mean (Mathematical Model)}

Start stating the concept of fuzzy c-means clustering problem, then precisely explain algorithm of FCM clustering [3].

Definition 1: "The fuzzy c-means clustering problem". A set of data on $n$ points have been given with $d$ features labeled by $n \times d$ matrix $\mathrm{X}, c$ is a positive integer considered as the number of clusters, while $m>1$ is the fuzzy constant, then find the partition matrix $U \in \mathrm{R}^{c^{\times n}}$ and centers of clusters centers $\mathrm{V}=\{\mathrm{v} 1, \mathrm{v} 2, \ldots, \mathrm{v} c\}$, such that:

$$
(U, V)=\arg \min _{U, V} \sum_{i=1}^{c} \sum_{j=1}^{n} u_{i j}^{m}\left\|x_{j}-v_{i}\right\|^{2}
$$

Herein, $\|\cdot\|$ indicates norm, ordinarily Euclidean norm. The partition matrix item $u i j$ indicate to membership of point $j$ in the cluster $i$. Furthermore, for each $j \in[1, \mathrm{n}]$, $\sum_{i=1}^{c} u_{i j}=1$ The objective function is defined as:

$$
\sum_{i=1}^{c} \sum_{j=1}^{n} u_{i j}^{m}\left\|x_{j}-v_{i}\right\|^{2} \triangleq o b j
$$

First, FCM algorithm calculates membership degrees by distances among cluster centers and points, then updates each cluster center according to the degree of membership. A solution is achieved through the computation of cluster centers and the iterative partition matrix. It would always be remembered that the clustering FCM only achieved a locally optimal solution, as well as initialization effect on the final outcome of clustering [3][4][8][13][5][6] . Detailed FCM clustering method is shown as below.

Input: data set $\mathrm{X}$ (an $n \times d$ matrix), number of clusters $c$, fuzzy constant $m$.

Output: partition matrix $\mathrm{U}$, centers of clusters $\mathrm{V}$.

Initialize: sample $\mathrm{U}$ (or $\mathrm{V}$ ) randomly from proper space.

While $\mid$ objold - objnew $\mid 2>\varepsilon$, or not maximum number of iterations do

$$
\begin{gathered}
u_{i j}=\left[\sum_{k=1}^{c}\left(\frac{\left\|x_{j}-v_{i}\right\|}{\left\|x_{j}-v_{k}\right\|}\right)^{2 /(m-1)}\right]^{-1}, \forall \mathrm{i}, \mathrm{j} \\
v_{i}=\frac{\sum_{j=1}^{n}\left(u_{i j}\right)^{m} x_{j}}{\sum_{j=1}^{n}\left(u_{i j}\right)^{m}}, \mathrm{Vi} \\
o b j=\sum_{i=1}^{c} \sum_{j=1}^{n} u_{i j}^{m}\left\|x_{j}-v_{i}\right\|^{2}
\end{gathered}
$$




\section{$4 \quad$ Methodology}

Our system design scheme for typological analysis for dynamic Ad Hoc network (DANET) nodes movement involves three stages:

Stage 1-Data collection: Collection of data is obtained as the basic data for NS2 simulator as described in the next subsection. Typically, a high-dimensional dataset is created to obtain enough and rich of system information. Data clustering could be viewed such a "coarse" modeling based on information (or data structure) derived from data mines. The dynamic behaviour of the system should be sufficiently excited to data-clustering problem so that the data from the system are sufficiently abundant.

Stage 2-Dimension reduction: High-dimensional dataset, however, can result in the various processing system working inefficient, or ineffective. It is therefore beneficial firstly to reduce data dimension to a reasonable size, retain just as much as the original beneficial information as possible then feed the reduced dimension data into the clustering system.

Stage 3-Data clustering: Realizing the data clustering feature using FCM, to derive main spatial distribution aspect of spatiotemporal dynamic systems. FCM would be used for data analysis to identify the intrinsic spatial distribution nature of the spatiotemporal dynamic systems as described in subsection 4.2.

\subsection{Simulator and data set}

In this paper, a simulation system is required to build the proposed system as a dynamic ad hoc network (DANET) and collect data set as csv file. Network Simulator version two (NS2) is employed to simulate this system. Topology, mobility, traffic model, simulation behaviors has established at NS2. Nodes behaviors will design to generate real world of mobility nodes in DANET. The mobility model is increasingly dependent on different parameters of nodes as described in Table 1. Nodes parameters are the starting point of the location for mobile node, and node speed, velocity and direction modified a long time. Sensitive information, control data, notification messages, cooperative awareness messages and warning messages at the transport layer are based on UDP/TCP agent. These agents have the capability to generate the environment of nodes communication. some parameters of communication are sited, such as traffic and packet size. For establishing the traffic model for DANET, TCP transport protocol is employed. The mobility and traffic models are established to represent patterns of communication and movement of DANET. Hence, these models set their acceleration, velocity, location, and speed modified over time. Routing protocol utilized to facilitate communication within the network in the mobility and traffic model of network. So, it is essential to use Ad Hoc On Demand Distance Vector (AODV) routing protocol, some files of the AODV routing protocol must be modified in order to broadcast essential information about position of node between nodes, its direction, and speed of each node. 
Table 1. Parameters of Simulation

\begin{tabular}{|l|c|}
\hline \multicolumn{1}{|c|}{ Parameters } & Rate \\
\hline Simulation time & 200s \\
\hline Simulator & ns2.35 \\
\hline Number of nodes & 9 nodes \\
\hline Topology & $500 \times 500$ \\
\hline Transport Protocol & TCP \\
\hline Channel type & Wireless \\
\hline Routing Protocol & AODV \\
\hline Radio Propagation Mode & Two Ray Ground \\
\hline Queue Length & 50 packets \\
\hline Speed & $1 \mathrm{~m} / \mathrm{s}$ \\
\hline MAC protocol & IEEE 802.11 \\
\hline Network Interface type & Physical / Wireless \\
\hline
\end{tabular}

CSV file of NS2 is considered one of the most important output of the simulation systems of DANET as a collected data set file. The design steps of the simulated system are shown in Figure 1.

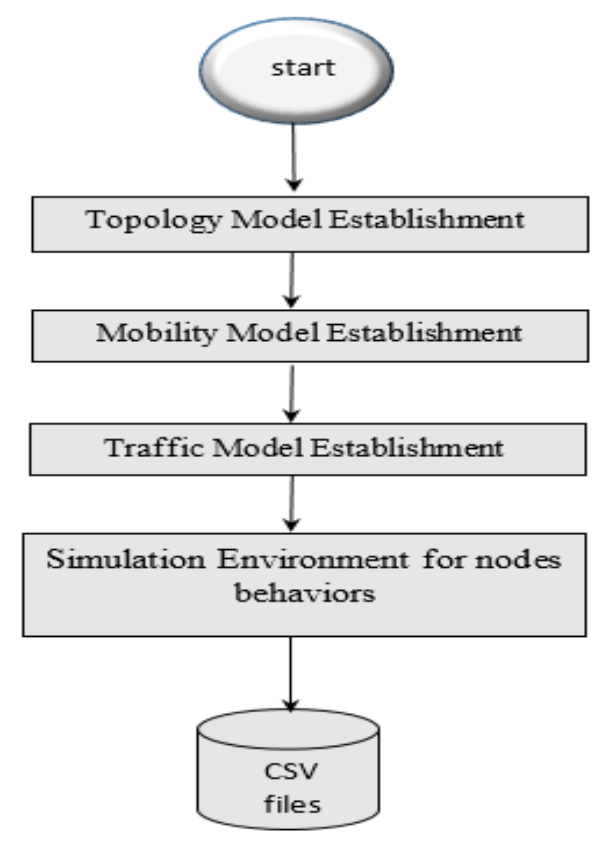

Fig. 1. A simulated system design steps

However, high-dimensional dataset has collected in the csv output file. So, first we reduced the dimension of the data keeps as much of useful original information such as position information to be the input data set to the clustering processing system. 


\subsection{Clustering by using FCM}

Well after reduction stage, the original high dimensional dataset assumed to be $\mathrm{D}$ reduced into a low dimensional dataset $\mathrm{S}=\{\mathrm{s} 1, \mathrm{~s} 2, \ldots, \mathrm{sN}\}$, with $\mathrm{Sj}=(\mathrm{sj} 1, \ldots, \mathrm{sjp})$ where sj will be the jth pattern $(1 \leq \mathrm{j} \leq \mathrm{N})$, sji be the ith feature of the jth pattern- $(1 \leq$ $\mathrm{i} \leq \mathrm{p}$.), the patterns number $\mathrm{N}, \mathrm{p}(\mathrm{p}<\mathrm{P})$ such as $\mathrm{P}$ the number of original dimensionality features, and $\mathrm{p}$ the number of features with reduced dimensionality.

FCM presented in Section 3 will be executed directly on S. Utilizing three FCM steps mentioned above, we get the matrix of fuzzy-partition $U=[\mu \mathrm{kj}] \mathrm{c} \times \mathrm{N}$ as well as matrix of cluster center $\mathrm{V}=[\mathrm{v} 1, \ldots, \mathrm{vc}]$, and finally, find grouping of data. It should also remember the $\mathrm{U}$ and $\mathrm{V}$ initialization can affect the results of the clustering. The algorithm can be formulated as a sequence of iterations under the required conditions $\mathrm{U}$ and V. An appropriate number of clusters upper bound must be selected Cmax. Also, the fuzziness factor $\mathrm{m}>1$ and the termination criteria must be set.

Following the initialization step; the algorithm proceeds successively with the partition matrix and prototype updates until a predefined termination criterion has been reached. The algorithm will halt while neither major updates in the partition matrix, nor changes in the prototypes, nor cost function improvements.

The following are the algorithmic procedure of FCM clustering technique to obtain the results as shown in the experimental section:

1. Initialization of the input parameters such as the dataset matrix [no. of patterns $\times$ no. of features], no. of clusters, fuzzy constant, initial values of partition matrix as zeros', and initial values of centers matrix from the initial implementation of the algorithm.

2. While neither major updates in the partition and centers matrix do 3 and 4.

3. Find updated partition matrix by implementing the equation (3).

4. Find updated centers matrix by implementing the equation (4).

5. Until the criteria of the equation (5) is satisfied.

\section{Experiment Results and Discussion}

The objectives of the experiment are to demonstrate the proposed clustering technique as being able to provide various rational clusters in different granulations and perspectives, as well as to display how the principle of fuzziness can be applied to DANET data sets. FCM has been implemented as a tool to apply and check data set behaviour. The cluster centers and membership matrix are updated within each iteration. Initial cluster centers are randomly selected to allow various outcomes to be obtained and not to be stuck to a certain one. The number of iterations in the case of divergence reaches the maximum permissible iterations' number and the algorithm is terminated.

Popular metrics are used to performance evaluations of clustering algorithms like cluster center stability, changes of cluster center, cluster stability average, node stability, changes of node, average node stability etc. These are quite generic terms with DANET specifications that are needed to provide consistency between various nodes. 
The interconnection and effects between nodes should be analyzed and presented as well. Test scenarios with different node movement patterns should be provided.

The initial parameters for testing the results of centers and nodes behaviors as follows: Number of centers $=4, \mathrm{~m}=2$, number of patterns (for dynamic nodes) $=1818$ (for 9 nodes), number of features $=2$ (x, y position coordinate values) for each pattern, time $(1 \ldots n) n=200 \mathrm{~s}$ the time of simulation.

For the first iteration with the initial centers values [90.094002, $158.88038400000002], \quad[98.645476,134.96348600000002],[45.0, \quad 285.0]$, [85.674718, 171.240306]], the initial result is shown as follows: Figures 2,3,4,5 and Table 2.

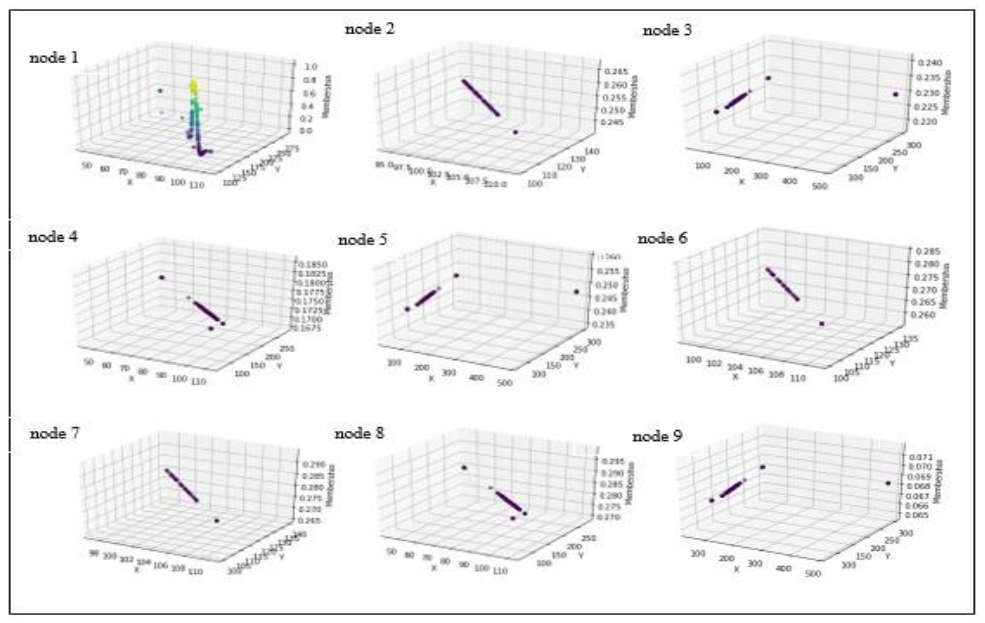

Fig. 2. Nodes behavior related to center 1 at the first iteration

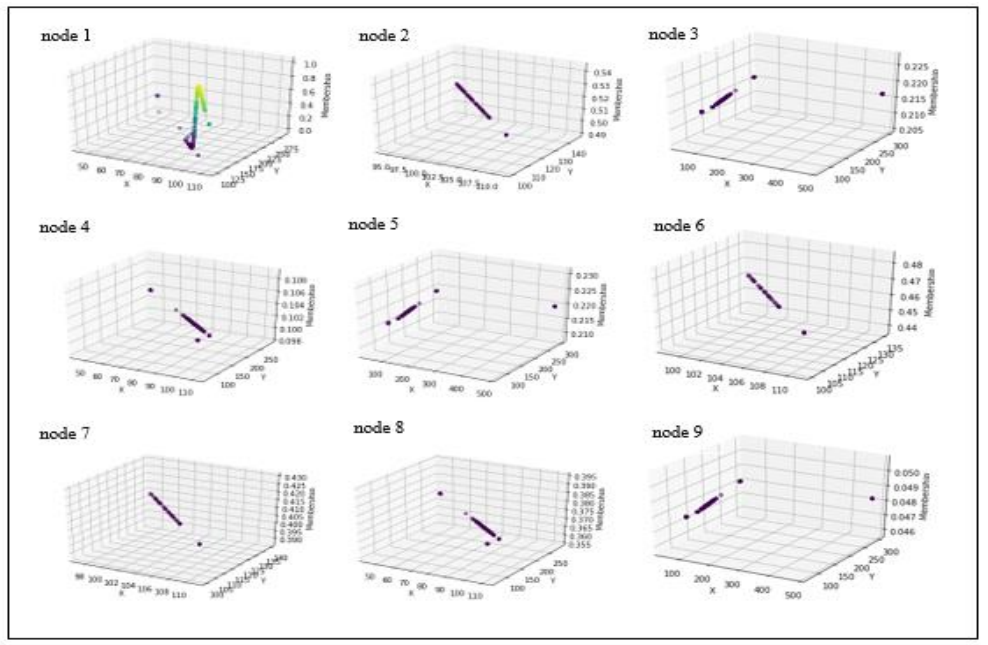

Fig. 3. Nodes behavior related to center 2 at the first iteration 


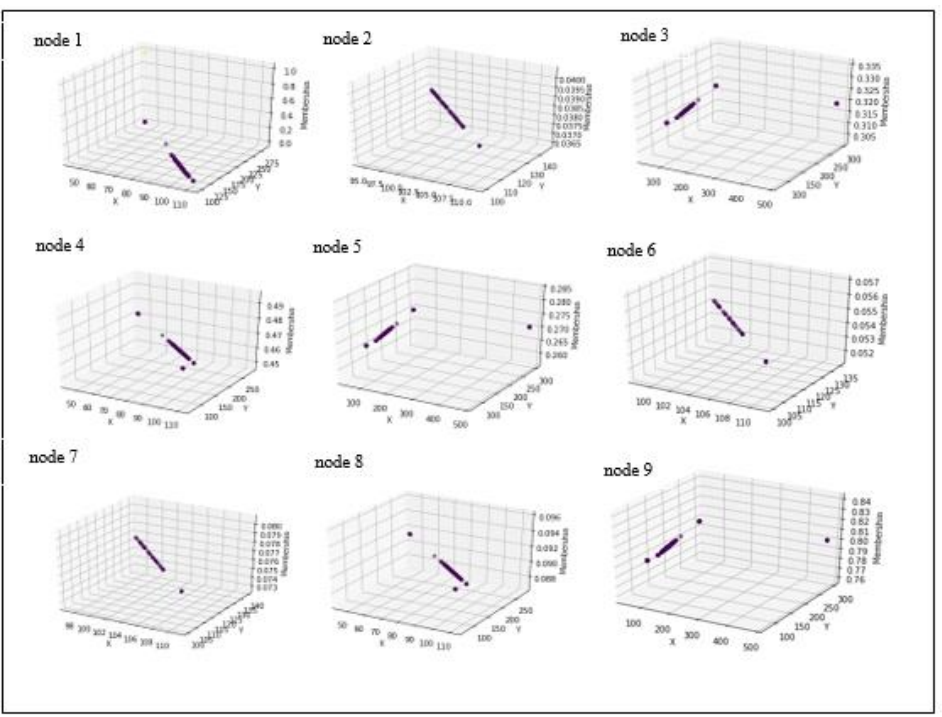

Fig. 4. Nodes behavior related to center 3 at the first iteration

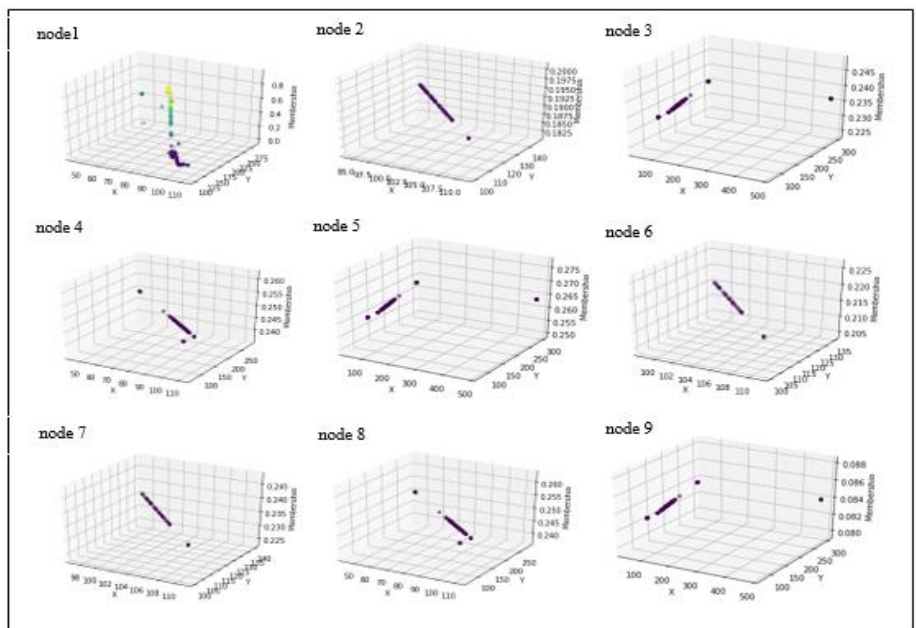

Fig. 5. Nodes behavior related to center 4 at the first iteration 
Table 2. Results of the first iteration with the initial centers values

\begin{tabular}{|c|c|c|c|c|}
\hline $\begin{array}{c}\text { Nodes at } \\
\text { time t1 }\end{array}$ & Center1 Membership & Center2 Membership & Center3 Membership & Center4 Membership \\
\cline { 1 - 4 } $\begin{array}{c}\text { Nodes } \boldsymbol{a t} \\
\text { time } \boldsymbol{n}\end{array}$ & & & & \\
\hline 1 & 0.21487517873497625 & 0.6173317043705212 & 0.02148911861838311 & 0.14630399827611945 \\
\hline 1 & 0.3406342870674682 & 0.24069092215553115 & 0.0 & 0.4186747907770006 \\
\hline 2 & 0.2542320544104734 & 0.5165653821717846 & 0.03837631858876760 & 0.19082624482897437 \\
\hline 2 & 0.2542320544104734 & 0.5165653821717846 & 0.03837631858876760 & 0.19082624482897437 \\
\hline 3 & 0.22895486498076237 & 0.21625874629098926 & 0.31882751052276004 & 0.23595887820548833 \\
\hline 3 & 0.22895486498076237 & 0.21625874629098926 & 0.31882751052276004 & 0.23595887820548833 \\
\hline 4 & 0.17645594105999077 & 0.10350386340388383 & 0.4711616967877681 & 0.2488784987483574 \\
\hline 4 & 0.17645594105999077 & 0.10350386340388383 & 0.4711616967877681 & 0.2488784987483574 \\
\hline 5 & 0.24701379226018244 & 0.21934011107306636 & 0.27023076819277514 & 0.26341532847397603 \\
\hline 5 & 0.24701379226018244 & 0.21934011107306636 & 0.27023076819277514 & 0.26341532847397603 \\
\hline 6 & 0.27024183094810905 & 0.4605031498511551 & 0.0541655173693444 & 0.21508950183139136 \\
\hline 6 & 0.27024183094810905 & 0.4605031498511551 & 0.0541655173693444 & 0.21508950183139136 \\
\hline 7 & 0.27949884399724906 & 0.4083651086897013 & 0.07656180656856496 & 0.23557424074448463 \\
\hline 7 & 0.27949884399724906 & 0.4083651086897013 & 0.07656180656856496 & 0.23557424074448463 \\
\hline 8 & 0.28422397939000205 & 0.3747770725109858 & 0.09122819255597979 & 0.24977075554303238 \\
\hline 8 & 0.28422397939000205 & 0.3747770725109858 & 0.09122819255597979 & 0.24977075554303238 \\
\hline 9 & 0.06820658505896916 & 0.04819451968929764 & 0.7997659435690931 & 0.08383295168264007 \\
\hline & & 5 & & \\
\hline 9 & 0.06820658505896916 & 0.04819451968929764 & 0.7997659435690931 & 0.08383295168264007 \\
\hline & & 5 & & \\
\hline & & & & \\
\hline & & & & \\
\hline & & & & \\
\hline
\end{tabular}

After the first iteration, ten times of iteration implemented to obtain the best results and reach the maximum values of stability for both cluster centers and nodes $(98.41$ $\%, 99.99 \%$ ) respectively at the last one as shown in Tables 4,5 and Figures 10, 11.

The results of the last iteration with the updated centers values [[479.9289455172455, 299.9710722081992], [73.79966176098901, 92.97903317561867], [32.72817691173006, 241.54349689141256], [249.48095286383295, 249.6910117429727]] is shown in Figures 6,7, 8, 9 and Table 3. 


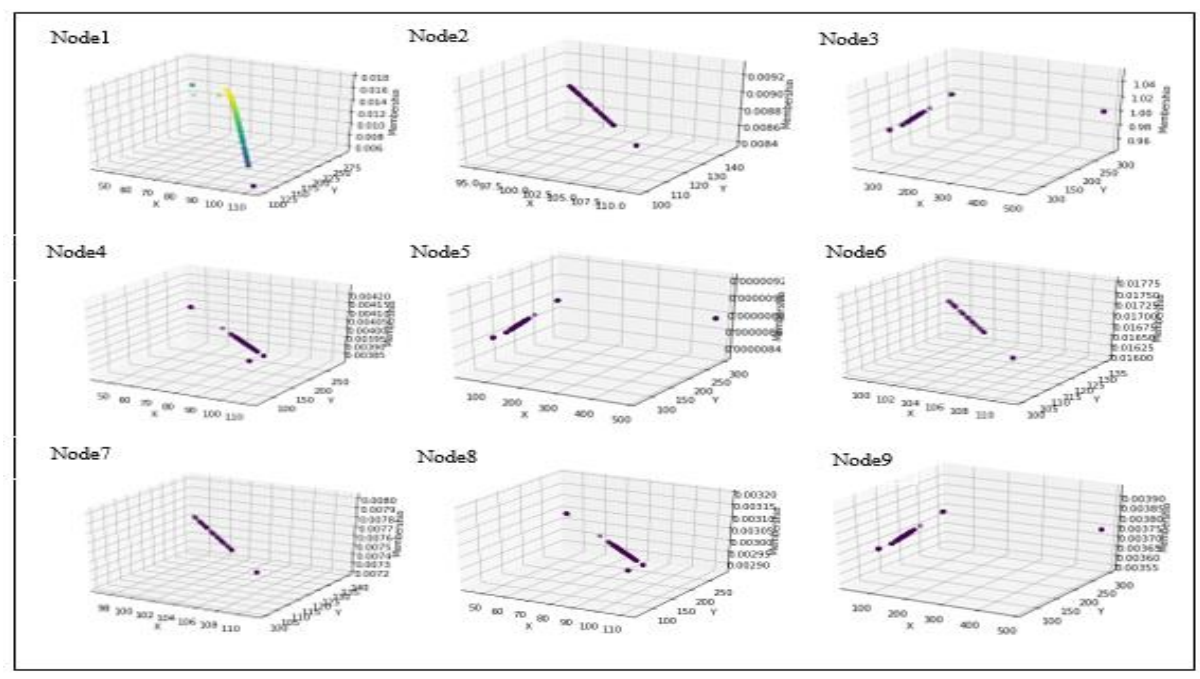

Fig. 6. Nodes behavior related to center 1 at the last iteration

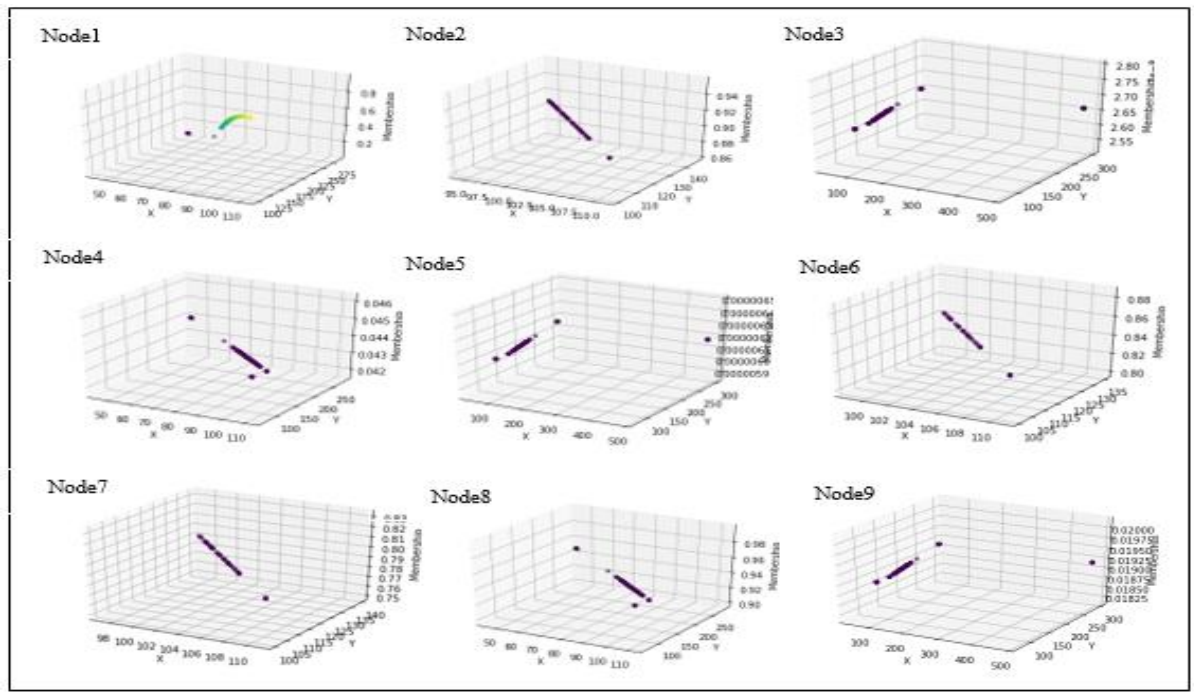

Fig. 7. Nodes behavior related to center 2 at the last iteration 


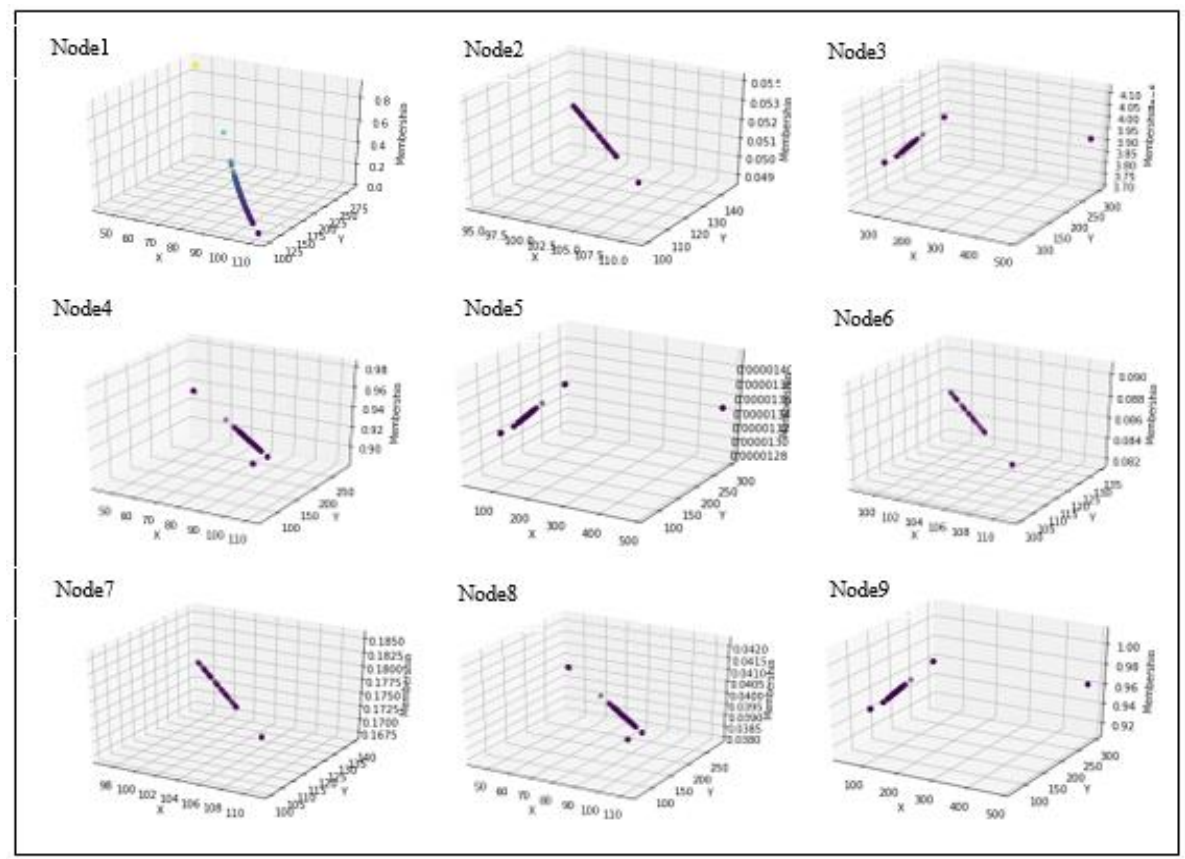

Fig. 8. Nodes behavior related to center 3 at the last iteration

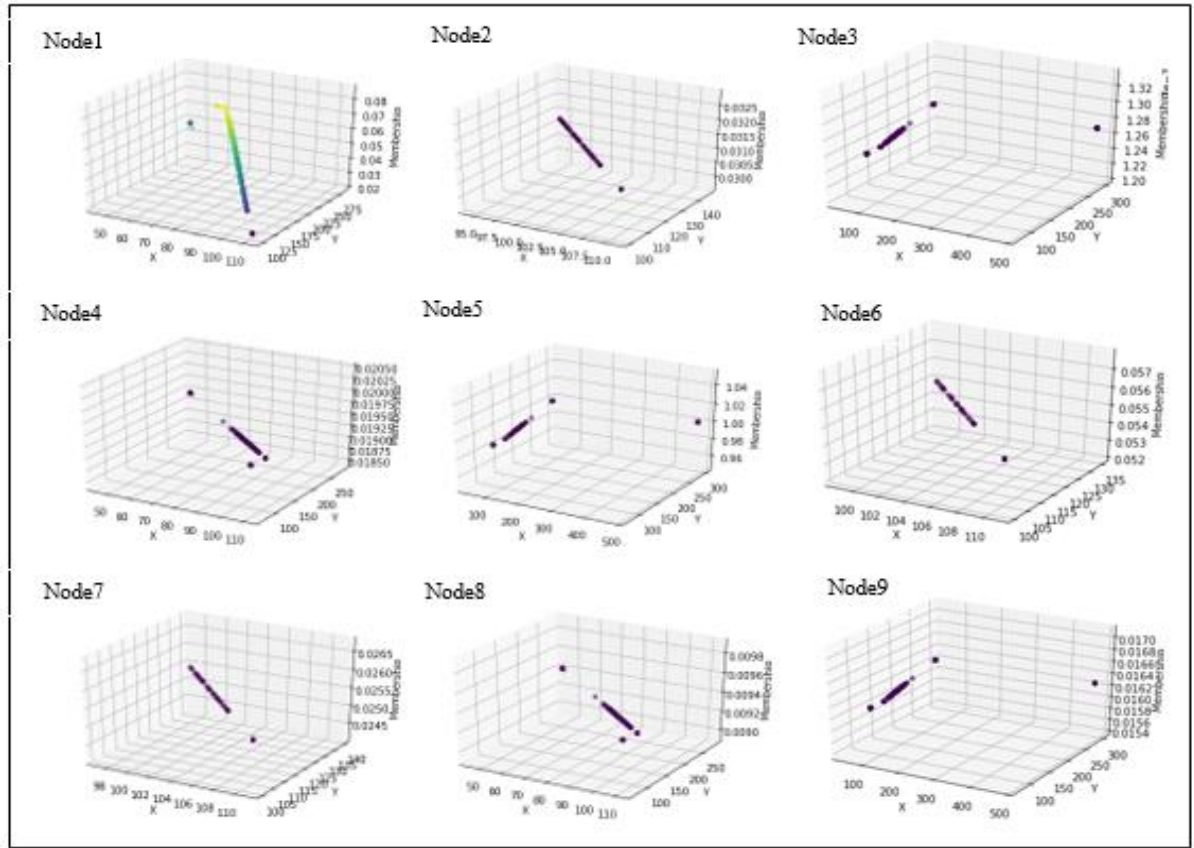

Fig. 9. Nodes behavior related to center 4 at the last iteration 
Table 3. Results of the last iteration with the final updated centers values

\begin{tabular}{|c|c|c|c|c|}
\hline $\begin{array}{c}\text { Nodes at } \\
\text { time t1 }\end{array}$ & \multirow{2}{*}{ Center1 Membership } & \multirow{2}{*}{ Center2 Membership } & \multirow{2}{*}{ Center3 Membership } & \multirow{2}{*}{ Center4 Membership } \\
\hline $\begin{array}{c}\text { Nodes at } \\
\text { time n }\end{array}$ & & & & \\
\hline 1 & $\begin{array}{c}0.00577478021669512 \\
5\end{array}$ & 0.932856169648133 & 0.03882413800330995 & 0.02254491213186207 \\
\hline 1 & $\begin{array}{c}0.01354168210069897 \\
8\end{array}$ & $\begin{array}{c}0.05621152286174308 \\
6\end{array}$ & 0.8825770627764558 & 0.04766973226110215 \\
\hline 2 & $\begin{array}{c}0.00884141333741526 \\
3\end{array}$ & 0.9085289030994165 & 0.0513955135267773 & $\begin{array}{c}0.03123417003639096 \\
2\end{array}$ \\
\hline 2 & $\begin{array}{c}0.00884141333741526 \\
3\end{array}$ & 0.9085289030994165 & 0.0513955135267773 & $\begin{array}{c}0.03123417003639096 \\
2\end{array}$ \\
\hline 3 & 0.999999807576452 & $\begin{array}{c}2.658345785778084 \mathrm{e}- \\
08\end{array}$ & $\begin{array}{c}3.90851139657537 \mathrm{e}- \\
08\end{array}$ & $\begin{array}{c}1.2675497619158333 \mathrm{e} \\
-07\end{array}$ \\
\hline 3 & 0.999999807576452 & $\begin{array}{c}2.658345785778084 \mathrm{e}- \\
08\end{array}$ & $\begin{array}{c}3.90851139657537 \mathrm{e}- \\
08\end{array}$ & $\begin{array}{c}1.2675497619158333 \mathrm{e} \\
-07\end{array}$ \\
\hline 4 & $\begin{array}{c}0.00402235865044590 \\
7\end{array}$ & $\begin{array}{c}0.04392145322296147 \\
5\end{array}$ & 0.9326090004214458 & $\begin{array}{c}0.01944718770514702 \\
2\end{array}$ \\
\hline 4 & $\begin{array}{c}0.00402235865044590 \\
7\end{array}$ & $\begin{array}{c}0.04392145322296147 \\
5\end{array}$ & 0.9326090004214458 & $\begin{array}{c}0.01944718770514702 \\
2 \\
\end{array}$ \\
\hline 5 & $\begin{array}{c}8.751442076483117 \mathrm{e}- \\
06\end{array}$ & $\begin{array}{c}\text { 6.174758158917101e- } \\
06\end{array}$ & $\begin{array}{c}1.3455918626396316 \mathrm{e} \\
-05\end{array}$ & 0.9999716178811381 \\
\hline 5 & $\begin{array}{c}8.751442076483117 \mathrm{e}- \\
06\end{array}$ & $\begin{array}{c}\text { 6.174758158917101e- } \\
06\end{array}$ & $\begin{array}{c}1.3455918626396316 \mathrm{e} \\
-05\end{array}$ & 0.9999716178811381 \\
\hline 6 & $\begin{array}{c}0.01687807871476704 \\
2\end{array}$ & 0.8420491375413669 & 0.08614557672969314 & $\begin{array}{c}0.05492720701417299 \\
5\end{array}$ \\
\hline 6 & $\begin{array}{c}0.01687807871476704 \\
2\end{array}$ & 0.8420491375413669 & 0.08614557672969314 & $\begin{array}{c}0.05492720701417299 \\
5\end{array}$ \\
\hline 7 & $\begin{array}{c}0.00761144449907962 \\
3\end{array}$ & 0.7906942378389198 & 0.17626875992131924 & 0.02542555774068136 \\
\hline 7 & $\begin{array}{c}0.00761144449907962 \\
3 \\
\end{array}$ & 0.7906942378389198 & 0.17626875992131924 & 0.02542555774068136 \\
\hline 8 & $\begin{array}{c}0.00303437557610524 \\
9\end{array}$ & 0.9474794570799289 & $\begin{array}{c}0.04008233221746447 \\
5\end{array}$ & $\begin{array}{c}0.00940383512650153 \\
4\end{array}$ \\
\hline 8 & $\begin{array}{c}0.00303437557610524 \\
9 \\
\end{array}$ & 0.9474794570799289 & $\begin{array}{c}0.04008233221746447 \\
5 \\
\end{array}$ & $\begin{array}{c}0.00940383512650153 \\
4 \\
\end{array}$ \\
\hline 9 & $\begin{array}{c}0.00373913082777167 \\
65 \\
\end{array}$ & $\begin{array}{c}0.01916274328436921 \\
5 \\
\end{array}$ & 0.9608473147507742 & $\begin{array}{c}0.01625081113708484 \\
7 \\
\end{array}$ \\
\hline 9 & $\begin{array}{c}0.00373913082777167 \\
65\end{array}$ & $\begin{array}{c}0.01916274328436921 \\
5\end{array}$ & 0.9608473147507742 & \begin{tabular}{|c|}
0.01625081113708484 \\
7
\end{tabular} \\
\hline
\end{tabular}


Table 4. Stability ratio of nodes

\begin{tabular}{|c|c|c|}
\hline Node no. & Center no. & $\begin{array}{l}\text { Stability ratio from first to the } \\
\text { latest two iterations }\end{array}$ \\
\hline 1 & 3 & $\begin{array}{r}93.96808632475456 \\
99.97217069891341 \\
99.9937879399294 \\
\end{array}$ \\
\hline 2 & 2 & $\begin{array}{l}68.00549235594379 \\
99.99862855841353 \\
99.99953033353947 \\
\end{array}$ \\
\hline 3 & 1 & $\begin{array}{l}91.663604822540585 \\
100.00000060444884 \\
100.00000016053599 \\
\end{array}$ \\
\hline 4 & 3 & $\begin{array}{c}99.41326027252914 \\
99.99862855841353 \\
100.00046966646053 \\
\end{array}$ \\
\hline 5 & 4 & $\begin{array}{c}80.1371142350535 \\
99.99985290213242 \\
99.99996612658182 \\
\end{array}$ \\
\hline 6 & 2 & $\begin{array}{l}99.79098139633342 \\
99.99581628307021 \\
99.99893965597106 \\
\end{array}$ \\
\hline 7 & 2 & $\begin{array}{c}93.965224118747867 \\
99.93476135888067 \\
99.9868876228809 \\
\end{array}$ \\
\hline 8 & 2 & $\begin{array}{l}94.252271265702525 \\
99.92323322350634 \\
99.98158532519142 \\
\end{array}$ \\
\hline 9 & 3 & $\begin{array}{r}8.71815671846195 \\
99.94287100897784 \\
99.98818702446861 \\
\end{array}$ \\
\hline Average ratio of nodes stability & \multicolumn{2}{|c|}{99.994372650617688888888888888889} \\
\hline
\end{tabular}

Table 5. Stability ratio of centers

\begin{tabular}{|c|c|c|}
\hline \multirow{2}{*}{ Center no. } & \multicolumn{2}{|c|}{$\begin{array}{c}\text { Stability ratio } \\
\text { from first to the latest two iterations }\end{array}$} \\
\cline { 2 - 3 } & \multicolumn{2}{|c|}{ X } \\
\hline \multirow{2}{*}{1} & 23.47086742124456 & 75.20359796257322 \\
& 99.89298862526 & 99.947315142376 \\
& 99.97166075553 & 99.98636314671 \\
\hline \multirow{2}{*}{3} & 52.886139144399 & 92.02556234234899 \\
& 86.470609444764 & 89.021834383158 \\
& 96.753153881899 & 97.212862323593 \\
\hline 4 & 39.65122780634361 & 70.81644799756864 \\
& 92.0242433406571 & 84.210484928161 \\
& 98.4018587429255 & 96.66986191699 \\
\hline Average ratio of centers & 11.34050039880715 & 59.11875274181548 \\
stability & 98.778739986418 & 99.033893598916 \\
& 99.72164106914 & 99.7825294549 \\
\hline
\end{tabular}




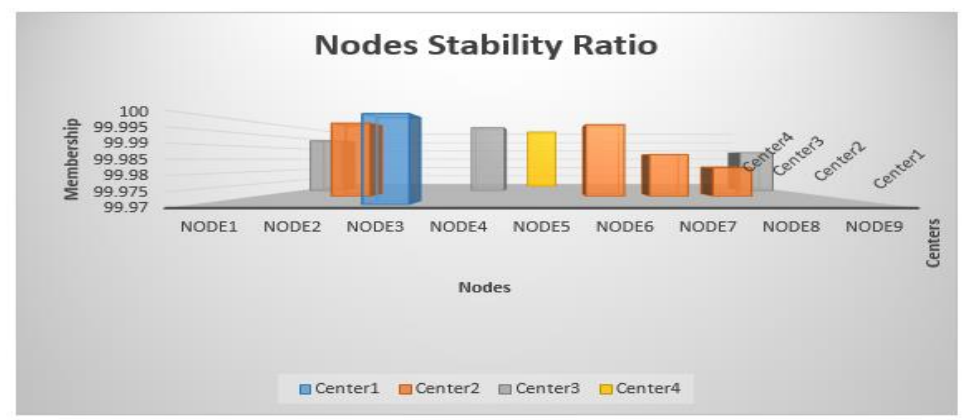

Fig. 10. Stability ratio of nodes

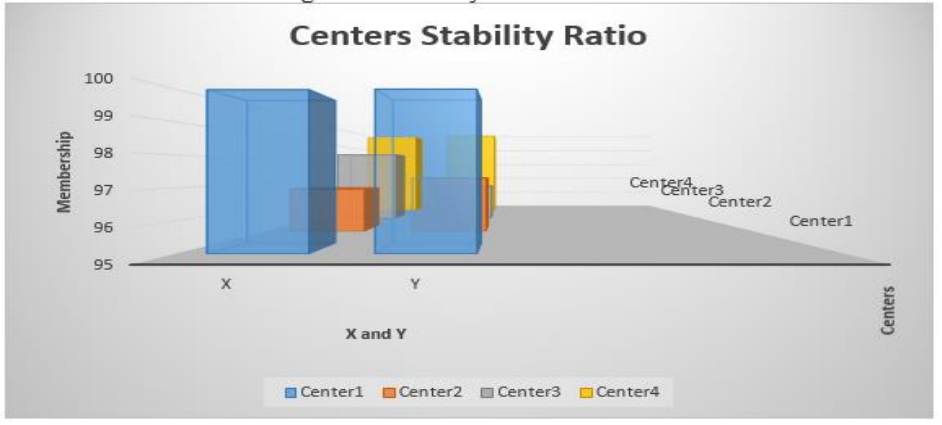

Fig. 11. Stability ratio of centers

So, to conclude the theoretic findings we introduce our clustering approach with the aim of increasing the stability of the network topology and making it less dynamic. This approach takes the position of nodes into consideration during the clustering process.

The stability occurs when the cluster centers is unable to perform the desired responsibilities or it leaves the cluster. The cluster centers' lifetime is a very significant aspect to examine the stability of the mechanism because if a cluster centers remains the head of a particular cluster for a long interval of time. So, we find the using of FCM algorithm provides more precise computation of the cluster membership by doing a full inverse-distance weighting, finding minimizers of objective function so it has been used successfully for DANET clustering applications. This technique achieves more efficient accuracy (stability) in the result. It will enhance the stability as well as reduce the computational cost caused by performing numerous computations to select centers after a short span of time (number of iterations) because it computes and updates the centers implicitly in precise and fast computation so we do not need another technique to improve the process. Improving stability and decreasing the overhead will increase the efficiency of the network.

We have evaluated our proposed method for accuracy (stability criteria) and efficiency (by comparing to other methods). 
However, we will perform extensive comparisons between existing clustering algorithms and our work to evaluate the performance (cluster stability) as shown in Table 6.

Table 6. Comparison between our method and previous methods

\begin{tabular}{|c|c|c|c|}
\hline $\begin{array}{c}\text { Reference } \\
\text { no. }\end{array}$ & Name of method & Simulation tool & Stability \\
\hline [14] & NA & $\mathrm{C}++$ & $34-46 \%$ \\
\hline$[15]$ & MobHiD & $\mathrm{NS} 2$ & Enhance the stability \\
\hline [16] & DGMA & $\mathrm{C}++$ & $30-60 \%$ \\
\hline [17] & AWCP-EWOA & NS-3 & $94.25 \%$ and $89.45 \%$ \\
\hline [18] & TCWCA & NA & Higher stability \\
\hline [19] & NA & $\mathrm{NS} 2$ & Higher stability \\
\hline$[20]$ & PB-WCA & $\mathrm{NS} 2$ & Increase stability \\
\hline [21] & OSCA & NS-3 & More stable \\
\hline$[22]$ & VLWBC & $\mathrm{NS} 2$ & $\begin{array}{l}\text { Improvement up to } 19 \% \text { in some } \\
\text { points which leads to higher } \\
\text { stability }\end{array}$ \\
\hline$[23]$ & NA & Java simulation & $\begin{array}{l}\text { Substantially improves cluster } \\
\text { stability }\end{array}$ \\
\hline$[24]$ & MOBIC & $\mathrm{NS} 2$ & $\begin{array}{l}\text { MOBIC yields a gain of close to } \\
33 \% \text { over Lowest-ID clustering }\end{array}$ \\
\hline [25] & VGBC & EstiNet 9.0 & Balancing and stability \\
\hline [26] & HBAC & EstiNet 8.1 & Efficient and stable \\
\hline [27] & StabTrust & OMNet++ & Stability level of 89 and 86 \\
\hline [28] & NA & MATLAB and C\# applications & $73 \%$ \\
\hline [29] & DCHA & $\mathrm{NS} 2$ & $36 \%$ \\
\hline$[30]$ & MPBC & $\begin{array}{l}\text { Open-source Matlabpackage wireless } \\
\text { network }\end{array}$ & $\begin{array}{l}\text { Substantially enhance the net- } \\
\text { work stability }\end{array}$ \\
\hline Our method & CDANM-FCM & NS2 and python Application & $99.99 \%$ \\
\hline
\end{tabular}

Many testing with different values of the fuzzy constant parameter (m) are implemented to show the best value to maximize the ratio of giving reasonable clusters. By the experiment, $2 \leq \mathrm{m}<3$ is the preferable range.

\section{Conclusion and Future Works}

The following points can be concluded from experimental results:

i. After ten times of iteration implemented, we have the best results and reach to the maximum values of stability for both cluster centres and nodes $(98.41 \%, 99.99 \%)$ respectively.

ii. Algorithm is superior with experiment in terms of stability convergence it will enhance the stability as well as reduce the computational cost caused by performing numerous computations to select centers after a short span of time (number of iterations).

iii. The FCM algorithm is successful in applying the fuzziness concept on DANET. 
iv. The behaviour of DANET's fuzzy c-means is proportionate to those of conventional numerical vector fuzzy c-means.

v. From the experimental results, the most appropriate value of $m$ was observed to be within the range: $2 \leq \mathrm{m}<3$.

vi. The principle of fuzziness is implemented, and the results obtained from the algorithm are given more significance and easier interpretation.

vii. The results from the presented method rely fundamentally on $\mathrm{m}$, initial state, and the similarity /dissimilarity metric used to compute distance among DANET nodes and centres.

viii. Besides small values of $\mathrm{m}$ close to 1.1 , the algorithm 's behaviour is almost identical to hard clustering, it can be noticed from the matrix of membership.

ix. Rising factor $m$ helps to increase the iterations number required to find a solution. It could be clarified as in range $2 \leq \mathrm{m}<3$, beside the high rate of variance among the values of a feature, the fuzzy memberships degrees allocated at the level of event to feature as well as pattern to cluster increases.

x. The structure of the DANET nodes and the similarity between the values of the feature raises iterations' number of required to achieve a solution.

As we see in this study, FCM is presented as a major technique to analyze the behavior and find stable clustering topology for DANET nodes movement. So for future work suggesting precise mathematical model of clustering result considering uncertainties is necessary for the better performance, finding a suitable initialization method for clusters; because Spatio-temporal dynamics data contain the spatial ordering feature, an appropriate strategy of initialization will be addressed to achieve better performance; such as determining optimal cluster numbers. Also, trail of real DANET movement of participating nodes in a restricted geographical area may be the first step towards the real-life testing of this technology.

\section{$7 \quad$ References}

[1] S. Ranganathan, K. Nakai, and C. Schonbach, Unsupervised Learning: Clustering. Elsevier, 2018.

[2] H. Q. Liu, L. C. Yang, Y. Zhang, and L. Wu, "A Position Sensitive Clustering Algorithm for VANET.," Int. J. Online Eng., vol. 10, no. 1, 2014.

[3] M. Ye, W. Liu, J. Wei, and X. Hu, "Fuzzy-means and cluster ensemble with random projection for big data clustering," Math. Probl. Eng., vol. 2016, 2016.

[4] J. Gu, L. Jiao, S. Yang, and J. Zhao, "Sparse learning based fuzzy c-means clustering," Knowledge-Based Syst., vol. 119, pp. 113-125, 2017. https://doi.org/10.1016/j.knosys.20 16.12 .006

[5] X.-X. Zhang, H.-X. Li, and C.-K. Qi, "Spatially constrained fuzzy-clustering-based sensor placement for spatiotemporal fuzzy-control system," IEEE Trans. Fuzzy Syst., vol. 18, no. 5, pp. 946-957, 2010. https://doi.org/10.1109/tfuzz.2010.2058810

[6] F. Santoso, M. A. Garratt, and S. G. Anavatti, "A self-learning TS-fuzzy system based on the C-means clustering technique for controlling the altitude of a hexacopter unmanned aerial vehicle," in 2017 International Conference on Advanced Mechatronics, Intelligent Manufacture, and Industrial Automation (ICAMIMIA), 2017, pp. 46-51. https://doi.org/ $\underline{10.1109 / \text { icamimia.2017.8387555 }}$ 
[7] G. Xiong, "Data Acquisition and Mining Algorithm of Car Networking under Big Data Background,” Int. J. Online Biomed. Eng., vol. 15, no. 01, pp. 4-17, 2019. https://doi.org/ 10.3991/ijoe.v15i01.9772

[8] R. Dutta, S. Gupta, and M. K. Das, "Low-energy adaptive unequal clustering protocol using fuzzy c-means in wireless sensor networks," Wirel. Pers. Commun., vol. 79, no. 2, pp. 1187-1209, 2014. https://doi.org/10.1007/s11277-014-1924-7.

[9] A. Choukri, Y. Hamzaoui, M. Amnai, and Y. Fakhri, "Classification Algorithm Based on Nodes Similarity for MANETs," Int. J. Online Biomed. Eng., vol. 15, no. 05, pp. 86-100, 2019. https://doi.org/10.3991/ijoe.v15i05.9742

[10] W. Zhenhua, H. Xiaodong, Z. Hong, and L. Chang'an, "Research on clustering strategy for wireless sensor network based on fuzzy theory," in Lecture Notes in Computer Science (including subseries Lecture Notes in Artificial Intelligence and Lecture Notes in Bioinformatics), 2007, vol. 4864 LNCS, pp. 596-604. https://doi.org/10.1007/978-3-540-770244_54

[11] G. Abdulsahib, N. Sulaiman, and O. I. Khalaf, "Improving Ad Hoc Network Behaviour Using Clustering Technique with NS2,” Int. J. Innov. Res. Comput. Commun. Eng., vol. 2, no. 10, pp. 5951-5966, 2014.

[12] D. Wang, W. Pedrycz, and Z. Li, “A Two-Phase Development of Fuzzy Rule-Based Model and Their Analysis," IEEE Access, vol. 7, pp. 80328-80341, 2019. https://doi.org/10.11 09/access.2019.2919739

[13] T. Mamatha and P. Aishwarya, "An Efficient Cluster based Routing Protocol using Hybrid FCM-Q LEACH for Vehicular Ad Hoc Networks,” Int. J. Appl. Eng. Res., vol. 14, no. 7, pp. 1604-1612, 2019.

[14] Z. Y. Rawashdeh and S. M. Mahmud, "A novel algorithm to form stable clusters in vehicular ad hoc networks on highways," Eurasip J. Wirel. Commun. Netw., vol. 2012, no. 1, p. 15, 2012. https://doi.org/10.1186/1687-1499-2012-15

[15] C. Konstantopoulos, D. Gavalas, and G. Pantziou, "Clustering in mobile ad hoc networks through neighborhood stability-based mobility prediction," Comput. Networks, vol. 52, no. 9, pp. 1797-1824, 2008. https://doi.org/10.1016/j.comnet.2008.01.018

[16] Y. Zhang, J. M. Ng, and C. P. Low, "A distributed group mobility adaptive clustering algorithm for mobile ad hoc networks," Comput. Commun., vol. 32, no. 1, pp. 189-202, 2009. https://doi.org/10.1016/j.comcom.2008.10.002

[17] S. R. Valayapalayam Kittusamy, M. Elhoseny, and S. Kathiresan, "An enhanced whale optimization algorithm for vehicular communication networks," Int. J. Commun. Syst., p. e3953, 2019. https://doi.org/10.1002/dac.3953

[18] L. Shan and L. Zhang, "Research on Weighted Clustering Algorithm for Ad Hoc Networks Based on Topology Correlation," in Journal of Physics: Conference Series, 2019, vol. 1213, no. 3, p. 32024. https://doi.org/10.1088/1742-6596/1213/3/032024

[19] M. Ren, L. Khoukhi, H. Labiod, J. Zhang, and V. Veque, "A mobility-based scheme for dynamic clustering in vehicular ad-hoc networks (VANETs)," Veh. Commun., vol. 9, pp. 233-241, 2017. https://doi.org/10.1016/j.vehcom.2016.12.003

[20] S. Pathak and S. Jain, "A priority-based weighted clustering algorithm for mobile ad hoc network," Int. J. Commun. Networks Distrib. Syst., vol. 22, no. 3, pp. 313-328, 2019. $\underline{\text { https }}$ ://doi.org/10.1504/ijends.2019.10019381

[21] S. Pathak and S. Jain, "An optimized stable clustering algorithm for mobile ad hoc networks,” EURASIP J. Wirel. Commun. Netw., vol. 2017, no. 1, p. 51, 2017.

[22] A. Karimi, A. Afsharfarnia, F. Zarafshan, and S. A. R. Al-Haddad, "A novel clustering algorithm for mobile ad hoc networks based on determination of virtual links' weight to increase network stability," Sci. World J., vol. 2014, 2014. https://doi.org/10.1155/2014/432 $\underline{952}$ 
[23] M. Chatzidakis and S. Hadjiefthymiades, "Location aware clustering and epidemic trust management in mobile ad hoc network," in 2019 28th International Conference on Computer Communication and Networks (ICCCN), 2019, pp. 1-7. https://doi.org/10.1109/icc cn.2019.8847166

[24] P. Basu, N. Khan, and T. D. C. Little, "A mobility-based metric for clustering in mobile ad hoc networks," in Proceedings 21st International Conference on Distributed Computing Systems Workshops, 2001, pp. 413-418. https://doi.org/10.1109/cdcs.2001.918738

[25] M. Ahmad, A. A. Ikram, I. Wahid, F. Ullah, A. Ahmad, and F. A. Khan, "Optimized clustering in vehicular ad hoc networks based on honeybee and genetic algorithm for internet of things," Peer-to-Peer Netw. Appl., vol. 13, no. 2, pp. 532-547, 2020. https://doi.org/10. $1007 / \mathrm{s} 12083-019-00724-4$

[26] M. Ahmad, A. A. Ikram, R. Lela, I. Wahid, and R. Ulla, "Honeybee algorithm--based efficient cluster formation and optimization scheme in mobile ad hoc networks," Int. J. Distrib. Sens. Networks, vol. 13, no. 6, p. 1550147717716815, 2017. https://doi.org/10.11 77/1550147717716815

[27] K. A. Awan, I. Ud Din, A. Almogren, M. Guizani, and S. Khan, "StabTrust-A Stable and Centralized Trust-Based Clustering Mechanism for IoT Enabled Vehicular Ad-Hoc Networks," IEEE Access, vol. 8, pp. 21159-21177, 2020. https://doi.org/10.1109/access.2020. 2968948

[28] S. A. Sharifi and S. M. Babamir, "The clustering algorithm for efficient energy management in mobile ad-hoc networks," Comput. Networks, vol. 166, p. 106983, 2020. https:// doi.org/10.1016/j.comnet.2019.106983

[29] R. Sundar and A. Kathirvel, "Aggressively delivered mechanism over variable length density using a simulated annealing algorithm in mobile ad hoc network," Trans. Emerg. Telecommun. Technol., p. e3863, 2020. https://doi.org/10.1002/ett.3863

[30] M. Ni, Z. Zhong, and D. Zhao, "MPBC: A mobility prediction-based clustering scheme for ad hoc networks," IEEE Trans. Veh. Technol., vol. 60, no. 9, pp. 4549-4559, 2011. https:// doi.org/10.1109/tvt.2011.2172473

\section{Authors}

Sumaya Hamad is member of the College of Computer Science and Information Technology (CSIT) as Assist. Teacher at University of Anbar, Anbar, Iraq. received the B.Sc. (good) (first class) degree in computer science from University of Anbar, in 2002, and the M.Sc. degree in computer science from University of Anbar, in 2012. She is currently PhD student in Computer Science Department at University of Technology, Baghdad, Iraq. She has published 5 refereed journal and conference papers. Her current research interests include mobile computing, artificial intelligence, Ad Hoc networks, search engines, and information technology. Email: sumayah.hamad@uoanbar.edu.iq

Dr. Yossra H. Ali is Assistant Professor. She received her B.Sc., M.Sc. and PhD degrees in 1996, 2002 and 2006 respectively from Iraq, University of technology, department of Computer Sciences. She Joined the University of Technology, Iraq in 1997. During her postgraduate studies she worked on Computer Network, Information systems, Agent Programming and Image Processing, she has some experience in Artificial Intelligent and Computer Data Security, She Reviewer at many conference and journals, she supervision of undergraduate and postgraduate ( $\mathrm{PhD}$. and MSc.) dissertations for many students in Computer sciences, she has a number of profes- 
sional certificates, Yossra has published in well regarded journals. Email: 110017@uotechnology.edu.iq.

Shaimaa H. Shaker is an assistant professor of computer science at university of technology, Iraq. She received the master's degree from University of technology, Iraq 1996 and doctorate's degree from University of technology, Iraq 2006. Her researches mainly focused on Information Systems, Image processing and pattern recognition. She often works as Reviewer for international conferences and journals. Email: mailto:shaimaa.h.shaker@uotechnology.edu.iq.

Khattab M. Ali Alheeti received the B.Sc. Computer Science degree in 2000 from Baghdad, Iraq, M.Sc. (Hons.) Computer Networking and Information in 2009 from the University of Al al-Bayt, Jordan, and Ph.D. from Essex University in 2017, respectively. Currently, he is working at University of Anbar. Email: mailto:co.khattab.alheeti@uoanbar.edu.iq.

Article submitted 2020-06-05. Resubmitted 2020-07-29. Final acceptance 2020-07-30. Final version published as submitted by the authors. 